\title{
Nonepileptic seizures under levetiracetam therapy: a case report of forced normalization process
}

This article was published in the following Dove Press journal:

Neuropsychiatric Disease and Treatment

23 May 2014

Number of times this article has been viewed

\section{Francesca Anzellotti \\ Raffaella Franciotti \\ Holta Zhuzhuni \\ Aurelio D'Amico \\ Astrid Thomas \\ Marco Onofrj}

Department of Neuroscience and Imaging, Aging Research Centre, Gabriele d'Annunzio University Foundation, Gabriele d'Annunzio University, Chieti, Italy
Correspondence: Francesca Anzellotti Department of Neuroscience and Imaging, Aging Research Centre, Gabriele d'Annunzio University, 33 Via dei Vestini, Chieti 66013, Italy Tel +390871358525

Email f.anzellotti@unich.it
Abstract: Nonepileptic seizures (NES) apparently look like epileptic seizures, but are not associated with ictal electrical discharges in the brain. NES constitute one of the most important differential diagnoses of epilepsy. They have been recognized as a distinctive clinical phenomenon for centuries, and video/electroencephalogram monitoring has allowed clinicians to make near-certain diagnoses. NES are supposedly unrelated to organic brain lesions, and despite the preponderance of a psychiatric/psychological context, they may have an iatrogenic origin. We report a patient with NES precipitated by levetiracetam therapy; in this case, NES was observed during the disappearance of epileptiform discharges from the routine video/ electroencephalogram. We discuss the possible mechanisms underlying NES with regard to alternative psychoses associated with the phenomenon of the forced normalization process.

Keywords: nonepileptic seizures, forced normalization, levetiracetam, behavioral side effects

\section{Introduction}

Nonepileptic seizures (NES), also known as pseudoseizures or hysterical fits, ${ }^{1}$ are included in epileptic psychotic manifestations. NES are paroxysmal, time-limited episodes of abnormal behavior or obtunded consciousness, movements, sensations, and psychic states that may be mistaken for epilepsy, but are not accompanied by electroencephalogram (EEG) changes. In accordance with O'Hanlon et al, ${ }^{2}$ we prefer the acronym NES to the term pseudoseizures. NES are a common clinical problem for family physicians, internists, psychiatrists, neurologists, and neurosurgeons. This type of behavioral disturbance has a poorly defined epidemiology, reflecting difficulties in establishing its prevalence and incidence. Various estimates suggest that $5 \%-25 \%^{3}$ of patients being evaluated for epileptic seizures actually have NES, and thus NES are a significant health care problem. ${ }^{4}$ Although concepts concerning the etiology of NES are still in evolution, psychological explanations currently predominate: NES are considered a learned pattern of behavior due to environmental stressors. ${ }^{5}$ Improved diagnostic capabilities (especially video EEG) have shown that NES are more common than once believed, ${ }^{6}$ and are not exclusively associated with temporal lobe epilepsy. ${ }^{7,8}$ Patients with NES are often misdiagnosed as suffering from intractable epilepsy, and are thus potentially exposed to unnecessary anticonvulsant medications and other iatrogenic consequences. ${ }^{6}$

Although there is variability in the data, there is a general consensus that psychiatric disorders are more prevalent in patients with epilepsy than in the general population. ${ }^{7}$ Psychosis encompasses a broad and subtle mental condition. Common features include 
impaired content and coherence of thought, reduced connection to reality, hallucinations, delusions, disorganized speech and behavior, and extremes of affect and motivation. The detection of psychosis can be difficult, as many patients actively hide their aberrant behavior and delusional beliefs, and others are quietly psychotic, showing only quirky mannerisms. ${ }^{9}$ As in other chronic disorders, the prevalence rates of psychiatric symptoms in epilepsy vary widely among the different studies published in the literature, with higher prevalence among patients with poorly controlled seizures. ${ }^{9-12}$ Psychoses can occur during seizure freedom and during or after epileptic seizures. Epileptic psychoses include also the phenomenon of the forced normalization process (FN) and de novo psychosis following epilepsy surgery. FN is characterized by a subacute/acute onset of psychosis associated with dramatic reduction of epileptiform activity.

Neurologists and psychiatrists have debated the existence of the phenomenon of forced normalization since its description by Landolt in 1953, ${ }^{13}$ who introduced the concept with two cases who both developed personality and mood changes in association with normalization of their EEGs. Landolt ${ }^{13}$ defined FN as "the phenomenon characterized by the fact that, with the occurrence of psychotic states, the EEG becomes more normal or entirely normal, as compared with previous and subsequent EEG findings". The literature in the nineteenth century reveals a growing interest in this relationship, particularly in France and Germany, and there are clear descriptions of specific psychopathological states and epilepsy. ${ }^{14}$ At this time, such terms as epileptic equivalents, larval epilepsy, and transformed epilepsy seem to have crept into the literature, and generally indicate an alteration in the seizure status and/or the development of a behavioral disorder.

The emergence of new anticonvulsant drugs in the past decade and the increased reporting of behavioral disturbances with several of these drugs, associated with an improvement in seizure status, however, have brought FN again into the focus of scientific attention and curiosity. Patients taking ethosuximide, vigabatrin, levetiracetam (LEV), and topiramate (TPM) with multiple daily seizures (mostly if focal and originating from the limbic lobe), sleep disturbances, and previous psychiatric disorders seem to be more vulnerable. ${ }^{15} \mathrm{FN}$ has only been rarely reported in children and adolescents. ${ }^{16}$

We describe for the first time NES as expression of the FN process based on Krishnamoorthy and Trimble criteria (Table 1). ${ }^{17,18}$ The patient was taking TPM and LEV. NES after LEV administration has also been reported in a previous study. ${ }^{19}$
Table I Primary and supportive diagnostic criteria for FN

\section{Primary criteria}

I. Diagnosis of epilepsy supported by clinical history, EEG, and neuroimaging

2. Occurence of acute/subacute behavioral disturbances

3A. Reduction in the total number of epileptiform abnormalities counted in a $60-$ min awake EEG recording by over $50 \%$ compared to a similar recording performed during a normal state of behavior or

3B. Absence of seizures for at least one week corroborated by a relative or carer

Supportive criteria

I. Recent change (within 15 days) of the drug regimen

2. Similar behavioral disturbances in the past corroborated by a relative, carer, or general practitioner.

Note: To make the diagnosis of $\mathrm{FN}$, it is necessary to identify primary criteria I, 2 , and $3 \mathrm{~A}$ or primary criteria $\mathrm{I}, 2$, and $3 \mathrm{~B}$ and one supportive criterion.

Abbreviations: EEG, electroencephalography; FN, forced normalization.

\section{Case report}

A 53-year-old right-handed woman suffered from focal epilepsy, ${ }^{20}$ with seizures characterized by abrupt loss of consciousness and tonic and clonic phases. Her clinical history was significant for a family history of febrile seizures, a poor therapeutic compliance associated with recurrences of epileptic seizures, a diagnosis of fibromyalgia, and a recent reactive depression with psychosomatic symptoms and insomnia; the Hamilton rating scale assessed a score of moderate depression. She denied previous pseudoseizures, but her relatives referred to behavioral disturbances in the past (she presented anxiety and irritability often in seizure-free periods). Magnetic resonance imaging findings were normal. Interictal EEG abnormalities in left frontocentrotemporal channels were characterized by repetitive nonperiodic sharp waves at $100 \mathrm{mV}$ with reversal phase on $\mathrm{F} 7$ and sporadic anterior synchronous and asynchronous theta activity $(6-7 \mathrm{~Hz}$, 50-60 mV). Due to thrombocytopenia, previous treatment with valproic acid was progressively reduced with recurrences of seizures. Considering the obesity of the patient, TPM was titrated up to a total dose of $300 \mathrm{mg}$ /day. Good seizure control was obtained, but 3 months later the patient was admitted to our neurology clinic, due to abnormal behavior and confusion; she presented with spatial/temporal disorientation, she aimlessly moved her hands, and she was slowly turning her head right and left. She presented with postictal aphasia. Video EEG monitoring revealed incoming seizures with a left frontotemporal focus (Figure 1A; Supplementary video). She received $10 \mathrm{mg}$ of diazepam intravenously. The background EEG improved, showing generalized theta activities at a frequency of 6-7 Hz. Subsequently, her therapy was changed: LEV was added and titrated rapidly until 1,000 mg 

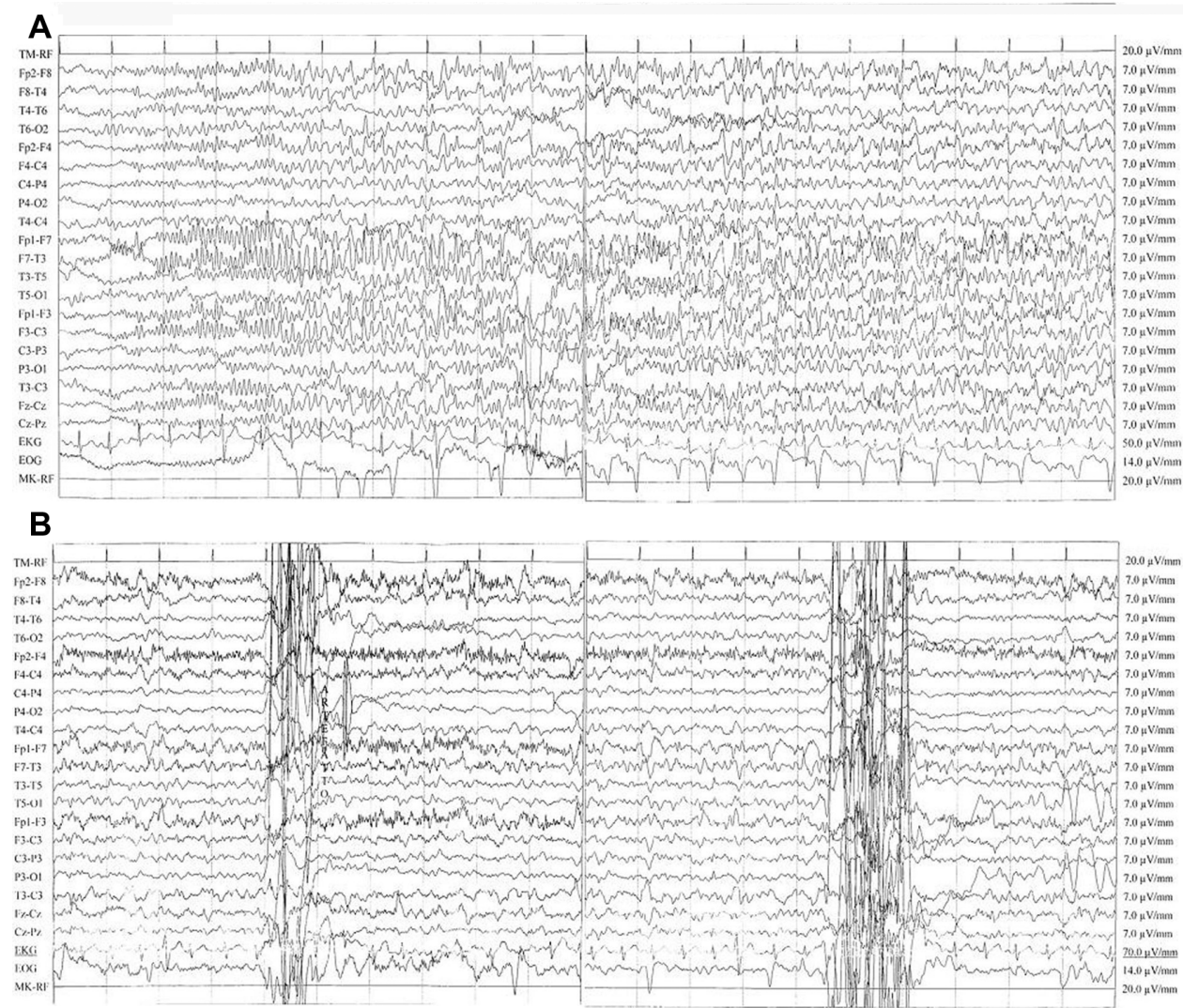

Figure I (A) Seizure with a left frontotemporal focus. Ictal electroencephalography (EEG) showed rhythmic and reluctant fast (I2-13 Hz) activity primarily involving the left frontotemporal area consisting of polyspikes of about $100 \mathrm{mV}$ amplitude with reversal phase in the F7 lead, then epileptic discharge involved all channels and showed a reduction in frequency $(6 \mathrm{~Hz}$ ). The patient was unconscious. Discharges consisting of high-amplitude sharp waves ( $90-100 \mu \mathrm{V})$ and slow waves (prominent on the frontotemporal areas) (high $30 \mathrm{~Hz}$, low 0.1 second; rate $15 \mathrm{~mm} / \mathrm{second}$ ). (B) EEG during pseudoseizures. Normal background activity with interictal abnormalities in left frontocentrotemporal channels: sporadic and nonperiodic sharp waves at $100 \mathrm{mV}$ with reversal phase on F7 and sporadic anterior synchronous and asynchronous theta activity $(6-7 \mathrm{~Hz}, 50-60 \mathrm{mV})$. Muscular artifacts on right frontal derivations and two abrupt movement artifacts were concomitant with fictitious spasms of the patient. No epileptic seizures were recorded. This recording showed a significant reduction of interictal activity in comparison with her previous EEGs.

twice daily. After 10 days, new episodes occurred: the patient appeared confused, she did not want to get out of bed, and when she answered our questions appropriately, she lamented about abdominal pain and asthenia. In addition, she had brief and repetitive tonic jerks of the trunk unaccompanied by loss of consciousness. During these episodes, which lasted about 50 seconds, her eyes were closed. Then she appeared drowsy for 10-15 minutes and could not recall these events. Immediately, EEG monitoring (Figure 1B) was performed, and it revealed no ictal abnormalities during these episodes, but only sporadic interictal sharp waves noted at baseline, supporting a diagnosis of NES. We observed a reduction of the epileptic activity in more than $50 \%$ of the waking EEG recording. The reduction of TPM to $100 \mathrm{mg}$ twice daily, the withdrawal of LEV and the introduction of lacosamide titrated to a dose of $300 \mathrm{mg} /$ day led to immediate resolution of NES. During follow-up examinations, neither seizures nor NES were reported anymore by patients and relatives.

\section{Discussion Nonepileptic seizures}

NES are classified by the Diagnostic and Statistical Manual of Mental Disorders (fourth edition) as a somatoform disorder. ${ }^{21}$ The condition is poorly understood, and is under-recognized by clinicians. The estimated incidence in an epilepsy outpatient clinic is $5 \%-25 \%{ }^{3}$ The correct diagnosis of NES, further complicated by the frequent coexistence of epilepsy, can be difficult if simply based on clinical criteria; a video EEG is always necessary. Several studies have described various semiological features of NES. ${ }^{22-24}$ Physical symptoms are suspected to result from psychosocial stress, and are only rarely intentional, as in malingering. NES are often suspected in patients with a history of somatization, abuse, or psychiatric comorbidity, ${ }^{25}$ or when the following clinical signs are identified: ${ }^{26}$ long duration, fluctuating course, asynchronous movements, pelvic thrusting, side-to-side head or body movement, ictal crying, memory recall, closed eyes, 
the uncommon ictal stuttering, and the "teddy-bear sign". Our knowledge of the clinical picture and context of NES has made only modest progress since Gowers ${ }^{27}$ summarized his understanding of "hysteroid" seizures in 1885, presenting criteria for distinguishing organic from inorganic seizures; at the end of the nineteenth century, Charcot was the first to describe "hysteroepilepsy" as a clinical disorder. ${ }^{28}$ The lack of consensus to define and categorize the illness underlies the complexity encountered in establishing effective treatment methodologies, and raises questions regarding the current diagnostic criteria. To address some of these issues, the broad expression "functional neurological disorder" is used for the Diagnostic and Statistical Manual of Mental Disorders (fifth edition). ${ }^{29}$ The etiology of NES is not entirely understood. Our case shows that it might have an iatrogenic origin, and could be resolved after drug discontinuation. LEV is an anticonvulsant with a favorable safety profile, but behavioral side effects are frequently reported, and were found to be independent of dose or seizure frequency. ${ }^{30}$ Possible mechanisms underlying behavioral disorders are idiosyncratic dose-unrelated drug effects, significantly increased by antiepileptic drugs, and alternative psychoses (or behavioral disturbances) associated with the phenomenon of FN. Dose-related toxicity and withdrawal syndromes have less importance. The existence of NES as a reversible drug-induced side effect has been identified. Barbiturates, benzodiazepines, and vigabatrin with significant $\gamma$-aminobutyric acid (GABA)-ergic properties and LEV with atypical mechanism of action produce behavioral side effects; NES are a behavioral problem. A case of NES appearing as an LEV side effect has previously been reported. $^{19}$

\section{Forced normalization}

There was a mutual antagonism between seizures and psychiatric problems: as the seizures improved, the psychiatric problem emerged. Neidermeyer et $\mathrm{al}^{1}$ suggested that preexisting "cerebral dysfunction" predisposes certain individuals to exhibit NES, and diffuse electroencephalographic slow-wave activity arising from anticonvulsant toxicity facilitates their expression. Since FN often occurs after an effective antiepileptic drug is added, the psychosis could be a side effect of medication, with EEG improvement an epiphenomenon. The concept of FN, first described by Landolt, ${ }^{13}$ refers to conditions where the disappearance of epileptiform discharges from the routine EEG is accompanied by some kind of behavioral disorder. The pathogenesis of this condition is debated. The most interesting hypotheses have been discussed by Wolf, ${ }^{31}$ who assumed that during "paradoxical normalization", a term that he preferred, the epilepsy is still active subcortically: the spread of discharge along unusual pathways is supposed to induce some of the acute psychotic symptoms. More recently, Bob ${ }^{32}$ analyzed the FN concept, hypothesizing that dissociative and somatoform symptoms mainly occur as a consequence of traumatic events. Even though FN has been recognized for a long time, the majority of published papers are case reports, probably as a result of the lack of validated diagnostic criteria. Only Krishnamoorthy and Trimble ${ }^{17}$ have proposed primary and supportive diagnostic criteria (Table 1), which have been revisited more recently. ${ }^{18}$ Ideally, such criteria should be tested, suitably modified if required, and adopted by scientific organizations that promote epilepsy research. Uniform diagnostic criteria such as these are the first step in systematic research. Our patient fulfills all the primary and supportive criteria; therefore, she was more susceptible to the occurrence of $\mathrm{FN}$, regardless of other provoking factors. Although a past history of psychosomatic symptoms and moderate depression are significant risk factors for NES, we showed the occurrence of behavioral abnormalities when a reduction of epileptic activity was observed in more than $50 \%$ of the waking EEG recording (compared to a similar recording performed during a normal state of behavior over 60 minutes) in a patient with an established diagnosis of epilepsy, with subacute onset of behavioral disturbance and a report of complete absence of seizures for at least 1 week (Table 1, Primary criteria). Furthermore the drug regimen was recently changed and parents referred to behavioral disturbances in the past (Table 1, Supportive criteria). Therefore, for the first time we have described and documented NES during a forced normalization process, pointing out that NES can be one of the multiple expressions of psychosis in FN. We obtained a resolution of the NES after LEV discontinuation, TPM reduction, and lacosamide introduction. For ethical reasons, we could not remove drug therapy to prove the disappearance of NES concomitant with the reappearance of the epileptic activity.

\section{Conclusion}

We hypothesized that in our case, FN was induced by LEV fast titration. Probably, lacosamide therapy controlled seizures without inducing FN, due to its different mechanism of action, although the impact of drug type and its mechanism of action is unclear. ${ }^{33}$ It is known that alterations in the balance of glutaminergic, dopaminergic, and GABA activity may cause seizures and behavioral disorder and can also play a role in the development of $\mathrm{FN} .{ }^{17}$ The emergence of new anticonvulsant drugs in the past decade and the increased reporting of behavioral disturbances with several 
of these drugs, associated with an improvement in seizure status, underlines the complexity of interaction between several potential pathogenic factors as the possible cause of FN. It is of interest that Landolt ${ }^{13}$ himself commented on the increase in the number of cases of FN with the introduction of succinimide drugs. Other researchers also observed this with barbiturates and hydantoins..$^{33}$ No data have reported behavioral side effects or FN cases with lacosamide, whereas both LEV and TPM might have induced NES: the patient had already been taking a stable dose of TPM for 3 months; therefore, it is likely that the psychotic episode could be ascribed to LEV introduction. In fact, during add-on therapy, we obtained EEG normalization. An interesting trial with LEV and $\mathrm{TPM}^{15}$ showed that some patients are prone to develop psychiatric drug-induced adverse events, regardless of pharmacological properties, and that an early limbic injury predisposes people to this type of psychiatric vulnerability. In our opinion, sudden seizure control obtained with fast titration and drug mechanism of action are in some selective cases the relevant factors for the development of psychosis in the FN process, while the association with a specific epilepsy syndrome may represent a bias connected to the probability of achieving complete seizure suppression: patients with symptomatic or multifocal epilepsy are less likely to be seizure-free than patients with idiopathic generalized epilepsy, which is usually associated with a better prognosis. Systematic research that identifies patients who meet Trimble's criteria in hospital-, institutional-, and community-based population groups, grafted onto modern techniques in molecular genetics and functional imaging, ${ }^{34}$ may well be the way forward in understanding this fascinating condition. Effective research in this area requires a combined attempt to establish international protocols and databases, while respecting patient privacy and rights.

\section{Disclosure}

The authors report no conflicts of interest in this work.

\section{References}

1. Neidermeyer E, Blumer D, Holscher E, Walker BA. Classical hysterical seizures facilitated by anticonvulsant toxicity. Psychiatr Clin (Basel). 1970;3:71-84.

2. O’Hanlon S, Liston R, Delanty N. Psychogenic nonepileptic seizures: time to abandon the term pseudoseizures. Arch Neurol. 2012;69: 1349-1350.

3. Szaflarski JP, Ficker DM, Cahill WT, Privitera MD. Four-year incidence of psychogenic nonepileptic seizures in adults in Hamilton County, $\mathrm{OH}$. Neurology. 2000;55:1561-1563.

4. Betts TA, Boden S. Pseudoseizures (non-epileptic attack disorder). In: Trimble M, editor. Women and Epilepsy. New York: Wiley; 1991: 243-258.
5. Ramani SV, Quesney LF, Olson D, Gumnit RJ. Diagnosis of hysterical seizures in epileptic patients. Am J Psychiatry. 1980;137:705-709.

6. Reuber M. Psychogenic nonepileptic seizures: Answers and questions. Epilepsy Behav. 2008;12:622-635.

7. Kogeorgos J, Fonagy P, Scott DF. Psychiatric symptom patterns of chronic epileptics attending a neurological clinic: a controlled investigation. Br J Psychiatry. 1982;140:236-243.

8. Kanner AM. Psychosis of epilepsy: a neurologist's perspective. Epilepsy Behav. 2000;1:219-227.

9. Adams SJ, O’Brien TJ, Lloyd J, Kilpatrick CJ, Salzberg MR, Velakoulis D. Neuropsychiatric morbidity in focal epilepsy. Br J Psychiatry. 2008;192:464-469.

10. Cockerell OC, Moriarty J, Trimble M, Sander JW, Shorvon SD. Acute psychological disorders in patients with epilepsy: a nation-wide study. Epilepsy Res. 1996;25:119-131.

11. Sperli F, Rentsch D, Despland PA, et al. Psychiatric comorbidity in patients evaluated for chronic epilepsy: a differential role of the right hemisphere? Eur Neurol. 2009;61:350-357.

12. Torta R, Keller R. Behavioral, psychotic, and anxiety disorders in epilepsy: etiology, clinical features, and therapeutic implications. Epilepsia. 1999;40 Suppl 10:S2-S20.

13. Landolt H. Serial EEG investigations during psychotic episodes in epileptic patients and during schizophrenic attacks. In: Lorentz De Haas AM, editor. Lectures on Epilepsy. Amsterdam: Elsevier; 1958: 91-133.

14. Schmitz B. Forced normalization: history of a concept. In: Trimble MR, Schmitz B, editors. Forced Normalization and Alternative Psychosis of Epilepsy. Petersfield, UK: Wrightson Biomedical; 1998:7-24.

15. Mula M, Trimble MR, Sander JW. Are psychiatric adverse events of antiepileptic drugs a unique entity? A study of topiramate and levetiracetam. Epilepsia. 2007;48:2322-2326.

16. Amir N, Gross-Tsur V. Paradoxical normalization in childhood epilepsy. Epilepsia. 1994;35:1060-1064.

17. Krishnamoorthy ES, Trimble MR. Forced normalization: clinical and therapeutic relevance. Epilepsia. 1999;40(10):57-64.

18. Krishnamoorthy ES, Trimble MR, Sander JW, Kannerc AM. Controversies in epilepsy and behavior: forced normalization at the interface between epilepsy and psychiatry. Epilepsy Behav. 2002;3:303-308.

19. Ignatenco A, Arzy S, Ghika J, et al. Nonepileptic seizures under levetiracetam therapy. Epilepsy Behav. 2010;19:526-527.

20. Berg AT, Berkovic SF, Brodie MJ, et al. Revised terminology and concepts for organization of seizures and epilepsies: report of the ILAE Commission on Classification and Terminology, 2005-2009. Epilepsia. 2010;51:676-685.

21. American Psychiatric Association. Diagnostic and Statistical Manual of Mental Disorders, fourth edition. American Psychiatric Press, Washington, DC, USA. 1994.

22. Chung SS, Gerber P, Kirlin KA. Ictal eye closure is a reliable indicator of psychogenic nonepileptic seizures. Neurology. 2006;66: 1730-1731.

23. DeToledo JC, Ramsay RE. Patterns of involvement of facial muscles during epileptic and nonepileptic events: review of 654 events. Neurology. 1996;47:621-625.

24. Vossler DG, Haltiner AM, Schepp SK, et al. Ictal stuttering: a sign suggestive of psychogenic nonepileptic seizures. Neurology. 2004;63: 516-519.

25. Anzellotti F, Franciotti R, Bonanni L, et al. Persistent genital arousal disorder associated with functional hyperconnectivity of an epileptic focus. Neuroscience. 2010;167:88-96.

26. Avbersek A, Sisodiya S. Does the primary literature provide support for clinical signs used to distinguish psychogenic nonepileptic seizures from epileptic seizures? J Neurol Neurosurg Psychiatry. 2010; 81:719-725

27. Gowers WR. Epilepsy and Other Chronic Diseases. New York: William Wood; 1885.

28. Havens LL. Charcot and hysteria. J Nerv Ment Dis. 1965;141:505-516. 
29. Stone J, LaFrance WC Jr, Brown R, Spiegel D, Levenson JL, Sharpe M. Conversion disorder: current problems and potential solutions for DSM-5. J Psychosom Res. 2011;71:369-376.

30. Helmstaedter C, Fritz NE, Kockelmann E, Kosanetzky N, Elger CE. Positive and negative psychotropic effects of levetiracetam. Epilepsy Behav. 2008; 13:535-541.

31. Wolf P. Acute behavioral symptomatology at disappearance of epileptiform EEG abnormality. Paradoxical or "forced" normalization. Adv Neurol. 1991;55:127-142.
32. Bob P. Dissociation, forced normalization and dynamic multi-stability of the brain. Neuro Endocrinol Lett. 2007;28:231-246.

33. Trimble MR. New antiepileptic drugs and psychopathology. Neuropsychobiology. 1998;38:149-151.

34. van der Kruijs SJ, Bodde NM, Vaessen MJ, et al. Functional connectivity of dissociation in patients with psychogenic non-epileptic seizures. J Neurol Neurosurg Psychiatry. 2012;83:239-247.

\section{Publish your work in this journal}

Neuropsychiatric Disease and Treatment is an international, peerreviewed journal of clinical therapeutics and pharmacology focusing on concise rapid reporting of clinical or pre-clinical studies on a range of neuropsychiatric and neurological disorders. This journal is indexed on PubMed Central, the 'PsycINFO' database and CAS.
The manuscript management system is completely online and includes a very quick and fair peer-review system, which is all easy to use. Visit http://www.dovepress.com/testimonials.php to read real quotes from published authors.

Submit your manuscript here: http://www.dovepress.com/neuropsychiatric-disease-and-treatment-journal 